\title{
CUT POINTS OF $X$ AND THE HYPERSPACE OF SUBCONTINUA $C(X)$
}

\author{
TOGO NISHIURA AND CHOON-JAI RHEE
}

\begin{abstract}
Let $X$ be a nondegenerate metric continuum and $p_{0}$ a point with $X=X_{1} \cup X_{2},\left\{p_{0}\right\}=X_{1} \cap X_{2}, X_{1}$ and $X_{2}$ continua. Denote by $C(X), C\left(X_{1}\right)$ and $C\left(X_{2}\right)$ the hyperspaces of nonempty subcontinua of $X, X_{1}$ and $X_{2}$ respectively.

THEOREM. $C(X)$ is contractible if and only if $C\left(X_{1}\right)$ and $C\left(X_{2}\right)$ are contractible and either $X_{1}$ or $X_{2}$ is contractible im kleinen at $p_{0}$ (a modification of connected im kleinen at $\left.p_{0}\right)$.

THEOREM. Let $X_{1}$ and $X_{2}$ satisfy Kelley's condition $K$. Then $C(X)$ is contractible when and only when either $X_{1}$ or $X_{2}$ is connected im kleinen at $p_{0}$.

Examples are given.
\end{abstract}

Let $X$ be a nondegenerate metric continuum and $p_{0}$ be a cut point of $X$. Denote by $X_{1}$ and $X_{2}$ subcontinua of $X$ such that $X=X_{1} \cup X_{2}$ and $X_{1} \cap X_{2}=\left\{p_{0}\right\}$. Each of $X, X_{1}$ and $X_{2}$ have their respective hyperspaces of nonempty subcontinua $C(X)$, $C\left(X_{1}\right)$ and $C\left(X_{2}\right)$ endowed with the Hausdorff metric $D$. In the present paper, a characterization of the contractibility of $C(X)$ is proved in terms of properties of the subcontinua $X_{1}$ and $X_{2}$. A corollary is then proved in which a characterization of the contractibility of $C(X)$ is established when both $X_{1}$ and $X_{2}$ have the property $K$ of [2]. Further applications of the main characterization theorem are also given.

Throughout the paper the symbol $I$ will be reserved for the closed interval $[0,1]$. For a general reference on $C(X)$, see [4].

1. The fibers of the cut point. By the fibers of the cut point $p_{0}$ we will mean the following closed subsets of $C(X)$.

$$
\begin{aligned}
& \mathscr{F}=\left\{A \in C(X) \mid p_{0} \in A\right\}, \\
& \mathscr{F}_{i}=\left\{A \in C\left(X_{i}\right) \mid p_{0} \in A\right\} \quad(i=1,2) .
\end{aligned}
$$

1.1. Proposition. $\mathscr{F}$ and $\mathscr{F}_{1} \times \mathscr{F}_{2}$ are homeomorphic. Hence $C(X)=C\left(X_{1}\right) \cup\left(\mathscr{F}_{1}\right.$ $\left.\times \mathscr{F}_{2}\right) \cup C\left(X_{2}\right)$ with the natural identifications.

Proof. Let $\Phi: \mathscr{F}_{1} \times \mathscr{F}_{2} \rightarrow 2^{X}\left(2^{X}=\right.$ space of nonempty closed subsets of $\left.X\right)$ be given by $\Phi\left(A_{1}, A_{2}\right)=A_{1} \cup A_{2}$. Clearly, $\Phi$ is continuous and into $\mathcal{F}$. If $A \in \mathcal{F}$ then $A_{1}=A \cap X_{1}$ and $A_{2}=A \cap X_{2}$ are connected because $p_{0}$ is a cut point of $X$.

Received by the editors July 27, 1979 and, in revised form, May 19, 1980; presented to the Society, January 5, 1980.

AMS (MOS) subject classifications (1970). Primary 54B20; Secondary 54D05, 54E40, 54F20.

Key words and phrases. Hyperspace of subcontinua $C(X)$, cut point, contractibility, connected im kleinen. 
Hence $\Phi$ is onto $\mathscr{F}$. To show $\Phi$ is one-to-one, let $\left(A_{1}, A_{2}\right)$ and $\left(B_{1}, B_{2}\right)$ be distinct elements of $\mathscr{F}_{1} \times \mathscr{F}_{2}$. We may suppose $A_{1} \neq B_{1}$. Since $A_{1} \cap B_{2}=\left\{p_{0}\right\}$ and $B_{1} \cap$ $A_{2}=\left\{p_{0}\right\}$, we have $A_{1} \cup A_{2} \neq B_{1} \cup B_{2}$. We now conclude $\Phi$ is a homeomorphism of $\mathscr{F}_{1} \times \mathscr{F}_{2}$ onto $\mathscr{F}$.

2. The first necesary condition. A contraction $h: C(X) \times I \rightarrow C(X)$ is called monotone if $h(A, t) \subset h\left(A, t^{\prime}\right)$ for $A \in C(X)$ and $t<t^{\prime}$.

2.1. Proposition [2]. If $C(X)$ is contractible then there is a monotone contraction $h: C(X) \times I \rightarrow C(X)$.

Proof. Let $\bar{h}: C(X) \times I \rightarrow C(X)$ be a contraction. Since $C(X)$ is arcwise connected, we may assume $\bar{h}(A, 1)=X$ for all $A \in C(X)$. For each $(A, t) \in C(X)$ $\times I$, define $h(A, t)=\bigcup\left\{\bar{h}\left(A, t^{\prime}\right): 0<t^{\prime} \leqslant t\right\}$. Then $h$ has the required property and is a contraction.

2.2. TheOREM. If $C(X)$ is contractible then so are $C\left(X_{1}\right)$ and $C\left(X_{2}\right)$.

Proof. Define $\gamma: X \rightarrow X_{i}(i=1$ or 2$)$ by

$$
\gamma(x)= \begin{cases}x, & \text { if } x \in X_{i}, \\ p_{0}, & \text { otherwise }\end{cases}
$$

and define $\bar{\gamma}: C(X) \rightarrow C\left(X_{i}\right)$ by $\bar{\gamma}(A)=\gamma[A]$ for all $A \in C(X)$. Then, $\bar{\gamma}$ is a retraction from $C(X)$ onto $C\left(X_{i}\right)$. Hence, since $C(X)$ is contractible, so is $C\left(X_{i}\right)$. This proof is the referee's and is somewhat different from our original proof.

3. Contractibility im kleinen. In order to discuss the second necessary condition we must define a notion related to connected im kleinen. (See [1] or [6] for the definition of connected im kleinen. The second reference uses the name locally connected.)

3.1. Definition. Let $Y$ be a metric space and $q_{0} \in Y$. We say $Y$ is contractible im kleinen at $q_{0}$ if for each $\varepsilon>0$ there are $\delta>0$ and continuous map $h_{\varepsilon}: Y \times I \rightarrow$ $C(Y)$ such that

(1) $h_{e}(q, 0)=\{q\}$ for each $q \in Y$;

(2) $d\left(q, q_{0}\right)<\delta$ implies $q_{0} \in h_{e}(q, 1)$;

(3) $d\left(q, q_{0}\right)<\delta$ and $t \in I$ imply diam $h_{e}(q, t)<\varepsilon$; and

(4) $h_{\varepsilon}(q, t) \subset h_{\varepsilon}\left(q, t^{\prime}\right)$ for $q \in Y$ and $t<t^{\prime}$.

3.2. Proposition. If $Y$ is contractible im kleinen at $q_{0}$ then $Y$ is connected im kleinen at $q_{0}$.

3.3. Definition [2]. A metric space $Y$ is said to have property $K$ if for each $\varepsilon>0$ there is $\delta>0$ such that whenever $a \in Y, a \in A \in C(Y)$ and $b \in Y$ with $d(a, b)$ $<\delta$ there is $B \in C(Y)$ with $b \in B$ and $D(A, B)<\varepsilon$.

It is proved in [2] that property $K$ implies $C(Y)$ is contractible for a continuum $Y$.

3.4. TheOREM. If a continuum $Y$ has property $K$ and is connected im kleinen at $q_{0}$ then it is contractible im kleinen at $q_{0}$. 
Proof. As in [2], there is a continuous function $\mu: C(Y) \rightarrow I$ such that $\mu(A)=0$ if and only if $A$ is a one-point set and $\mu(A)<\mu(B)$ if $A \subset B$ and $A \neq B$. It is proved in [2, pp. 23-24] with the aid of property $K$, that the map $h: Y \times I \rightarrow$ $C(Y)$, given by $h(q, t)=\cup\{A \mid q \in A$ and $\mu(A)<t\}$, is continuous. Let $\varepsilon>0$ be given. Then the set

$$
W=\left\{A \in C(Y) \mid \exists q_{1}, q_{2} \in A \ni d\left(q_{1}, q_{0}\right)>\varepsilon / 2 \text { and } d\left(q_{2}, q_{0}\right)<\varepsilon / 4\right\}
$$

is compact. Let $2 \lambda=\min \{\mu(A) \mid A \in W\}$. Then $\lambda>0$. Since $Y$ is connected im kleinen at $q_{0}$, there is a continuum $U$ with $q_{0}$ as an interior point such that $\mu(U)<\lambda$. Let $\delta>0$ be such that $d\left(q, q_{0}\right)<\delta$ implies $q \in U$. We may assume $\delta<\varepsilon / 4$. Then for $d\left(q, q_{0}\right)<\delta$ we have $q \in A \in C(Y)$ and $\mu(A)<\lambda$ implies $\operatorname{diam} A<\varepsilon$. Hence for $d\left(q, q_{0}\right)<\delta$ we have $q_{0} \in h(q, \lambda)$ and $\operatorname{diam} h(q, \lambda)<\varepsilon$. The proof is now easily completed.

3.5. Proposition. Let $Y$ be a continuum which is contractible im kleinen at $q_{0}$. Then for each $\varepsilon>0$ there are $\delta>0$ and continuous map $S_{\varepsilon}: Y \times I \rightarrow C(Y)$ such that

(1) $S_{e}(q, 0)=\{q\}$ for $q \in Y$;

(2) $q_{0} \in S_{e}(q, 1)$ for $d\left(q, q_{0}\right)<\delta / 2$;

(3) $\operatorname{diam} S_{\varepsilon}(q, t)<\varepsilon$ for $(q, t) \in Y \times I$;

(4) $S_{e}(q, t)=\{q\}$ for $d\left(q, q_{0}\right)>\delta$ and $t \in I$; and

(5) $S_{e}(q, t) \subset S_{e}\left(q, t^{\prime}\right)$ for $q \in Y$ and $t<t^{\prime}$.

Proof. Let $\varepsilon>0$. Then let $\delta$ and $h_{\varepsilon}$ be as in Definition 3.1. Define $\tau: Y \rightarrow \mathbf{R}$ as the continuous function

$$
\tau(q)=\left\{\begin{array}{l}
1, \quad d\left(q, q_{0}\right)<\delta / 2, \\
0, \quad d\left(q, q_{0}\right)>\delta, \\
2 \delta^{-1}\left(\delta-d\left(q, q_{0}\right)\right), \quad \delta>d\left(q, q_{0}\right)>\delta / 2 .
\end{array}\right.
$$

Define $S_{\varepsilon}: Y \times I \rightarrow C(Y)$ by

$$
S_{e}(q, t)= \begin{cases}h_{e}(q, t), & 0<t<\tau(q), \\ h_{e}(q, \tau(q)), & \tau(q)<t<1 .\end{cases}
$$

One verifies easily that $S_{\varepsilon}$ has the required properties.

3.6. Proposition. Let $Y$ be a continuum which is contractible im kleinen at $q_{0}$ and $\mathcal{G}=\left\{A \in C(Y) \mid q_{0} \in A\right\}$ be the fiber of $q_{0}$. Suppose $H: C(Y) \times I \rightarrow C(Y)$ is a continuous map such that $H(A, 0)=A, H(A, t) \subset H\left(A, t^{\prime}\right)$ for $t<t^{\prime}$ and $H(A, 1) \in$ $\mathcal{G}$ for $A \in C(Y)$. Then for each $\varepsilon>0$ there are $\eta>0$ and continuous map $H_{\varepsilon}$ : $C(Y) \times I \rightarrow C(Y)$ such that

(1) $0<\eta<\varepsilon$;

(2) $H_{e}(A, 0)=A$ for $A \in C(Y)$;

(3) $H_{e}(A, t) \subset H_{e}\left(A, t^{\prime}\right)$ for $A \in C(Y)$ and $t<t^{\prime}$;

(4) $H_{e}(A, 1) \in \mathcal{G}$ for $A \in C(Y)$;

(5) $D\left(H_{\varepsilon}(A, t), H_{e}(A, 1-\eta)\right)<\varepsilon$ for $D(A, \mathcal{G})>2 \eta$ and $1-\eta<t$;

(6) $H_{e}(A, t)=H(A, t)$ for $D(A, \mathcal{G})>2 \eta$ and $1-\eta>t$; 
(7) $D\left(H_{\varepsilon}(A, t), A\right)<\varepsilon$ for $D(A, \mathcal{G})<\eta$ and $t \in I$;

(8) $D\left(H_{e}(A, t), H_{e}(A, \alpha(A))\right)<\varepsilon$ for $\eta<D(A, \mathcal{G})<2 \eta$ and $1>t>\alpha(A)=$ $[(1-\eta)] / \eta(D(A, \mathcal{G})-\eta) ;$ and

(9) $H_{e}(A, t)=H(A, t)$ for $\eta<D(A, \mathcal{G})<2 \eta$ and $0<t<\alpha(A)=$ $[(1-\eta)] / \eta(D(A, \mathcal{G})-\eta)$.

Proof. Let $\varepsilon>0$ be given and let $\delta$ and $S_{\varepsilon}$ be given by Proposition 3.5. The set

$$
U=\left\{(A, t) \in C(Y) \times I \mid d\left(q_{0}, H(A, t)\right)<\delta / 2\right\}
$$

is open and contains $C(Y) \times\{1\} \cup \mathcal{G} \times I$, where $\mathcal{G}$ is the fiber of $q_{0}$. Let $\eta$ be such that

(i) $0<\eta<\varepsilon$;

(ii) $D(A, \mathcal{G})<\eta$ implies $(A, t) \in U$ for all $t \in I$; and

(iii) $1-2 \eta<t$ implies $(A, t) \in U$ for all $A \in C(Y)$.

Then the function

$$
\alpha(A)= \begin{cases}1-\eta, & D(A, \mathcal{G})>2 \eta, \\ 0, & D(A, \mathcal{G})<\eta, \\ \frac{1-\eta}{\eta}(D(A, \mathcal{G})-\eta), & \eta<D(A, \mathcal{G})<2 \eta,\end{cases}
$$

is continuous and $W=\{(A, t) \in C(Y) \times I \mid \alpha(A)<t<1\} \subset U$. For $\alpha(A)<t<$ 1 , let

$$
\beta(A, t)=(t-\alpha(A)) /(1-\alpha(A))
$$

and

$$
H(A, \alpha(A))_{t}=\bigcup\left\{S_{e}(q, \beta(A, t)) \mid q \in H(A, \alpha(A))\right\} .
$$

Then $\beta(A, t)$ and $H(A, \alpha(A))$, are continuous on the closed set $W$. Let $H_{\varepsilon}$ : $C(Y) \times I \rightarrow C(Y)$ be defined by

$$
H_{\varepsilon}(A, t)= \begin{cases}H(A, t), & 0<t<\alpha(A), \\ H(A, \alpha(A))_{t}, & \alpha(A)<t<1,\end{cases}
$$

where $A \in C(Y)$. Clearly, $H_{e}$ is continuous. Conditions (1)-(9) are easily verified.

3.7. Lemma. If $C(Y)$ is contractible and $Y$ is contractible im kleinen at $q_{0}$ then there is a deformation retract of $C(Y)$ onto the fiber $\mathcal{G}$ of $q_{0}$.

Proof. Let $H_{0}: C(Y) \times I \rightarrow C(Y)$ be a monotone contraction of $C(Y)$ and $0<\varepsilon_{1}<2^{-1}$. Then there are $\eta_{1}$ and $H_{\varepsilon_{1}}$ from Proposition 3.6. Proceeding inductively, we have sequences $\left\{\varepsilon_{n}\right\},\left\{\eta_{n}\right\}$ and $\left\{H_{\varepsilon_{n}}\right\}$ such that $0<\varepsilon_{n}<2^{-n}$ and $\eta_{n+1}$ and $H_{e_{n+1}}$ are related to $\varepsilon_{n}$ and $H_{\varepsilon_{n}}$ as in Proposition 3.6. Moreover, we may assume $\eta_{n}>\varepsilon_{n+1}$. One easily sees that the sequence $\left\{H_{e_{n}}\right\}$ converges uniformly on $C(Y) \times$ $I$, hence its limit $H$ is continuous. Also, it is easily verified that $H(A, 1) \in \mathcal{G}$ for $A \in C(Y)$ and $H(A, t)=A$ for $A \in \mathcal{G}$ and $t \in I$. An added consequence is $H(A, t) \subset H\left(A, t^{\prime}\right)$ for $t<t^{\prime}$. 
4. The second necessary condition. We return to $X=X_{1} \cup X_{2}$ with $X_{1} \cap X_{2}=$ $\left\{p_{0}\right\}$ as in $\S \S 2$ and 3 above. Suppose $h: C(X) \times I \rightarrow C(X)$ is a monotone contraction. Then $h\left(\left\{p_{0}\right\}, \cdot\right)$ maps $I$ into $\mathscr{F}_{1} \times \mathscr{F}_{2}$ and $h\left(\left\{p_{0}\right\}, 0\right)=\left(\left\{p_{0}\right\},\left\{p_{0}\right\}\right)$ and $h\left(\left\{p_{0}\right\}, 1\right)=\left(X_{1}, X_{2}\right)$. If $P_{i}: \mathscr{F}_{1} \times \mathscr{F}_{2} \rightarrow \mathscr{F}_{i}(i=1,2)$ are the natural projections then the functions

$$
s_{i}(t)=\operatorname{diam} P_{i}\left(h\left(\left\{p_{0}\right\}, t\right)\right) \quad(i=1,2)
$$

are continuous increasing functions with $s_{i}(0)=0$ and $s_{i}(1)>0(i=1,2)$. Let $t_{i}=\min \left\{t \mid s_{i}(t)>0\right\}(i=1,2)$.

4.1. Proposition. Suppose $t_{2}<t_{1}$. Then for each $\varepsilon>0$ there are $\delta>0$ and $\bar{t}$ such that

(1) $d\left(p, p_{0}\right)<\delta, p \in X_{1}$ and $0<t<\bar{t}$ imply diam $h(\{p\}, t)<\varepsilon$; and

(2) $d\left(p, p_{0}\right)<\delta$ and $p \in X_{1}$ imply $p_{0} \in h(\{p\}, \bar{t})$.

Proof. There is $\bar{t}$ such that $0<\operatorname{diam} h\left(\left\{p_{0}\right\}, \bar{t}\right)<\varepsilon / 2$. Let $A_{i}=X_{i} \cap h\left(\left\{p_{0}\right\}, \bar{t}\right)$ $(i=1,2)$. Then $\bar{t}>t_{2}$ and $\operatorname{diam} A_{2}>0$. Let $\bar{p} \in A_{2} \backslash\left\{p_{0}\right\}$ and $\eta$ be a positive number smaller than $d\left(\bar{p}, X_{1}\right)$. From the uniform continuity of $h$ there is $\delta>0$ such that $d\left(p, p_{0}\right)<\delta$ implies $D\left(h(\{p\}, t), h\left(\left\{p_{0}\right\}, t\right)\right)<\min \{\eta, \varepsilon / 4\}$ for $t \in I$. So, when $p \in X_{1}$ with $0<d\left(p, p_{0}\right)<\delta$ we have $h(\{p\}, \bar{t}) \backslash X_{1} \neq \varnothing$ and $h(\{p\}, \bar{t}) \backslash X_{2}$ $\neq \varnothing$. Hence $p_{0} \in h(\{p\}, \bar{t})$ for $d\left(p, p_{0}\right)<\delta$ and $p \in X_{1}$ and thereby (2) is proved. Finally for $p \in X_{1}, d\left(p, p_{0}\right)<\delta$ and $0<t<\bar{t}$ we have $\operatorname{diam} h(\{p\}, t)<$ $\operatorname{diam} h\left(\left\{p_{0}\right\}, t\right)+2 D\left(h(\{p\}, t), h\left(\left\{p_{0}\right\}, t\right)\right)<\operatorname{diam} h\left(\left\{p_{0}\right\}, \bar{t}\right)+\varepsilon / 2<\varepsilon$ and $(1)$ is proved.

4.2. Proposition. If $t_{2} \leqslant t_{1}$ then $X_{1}$ is contractible im kleinen at $p_{0}$.

Proof. Let $\bar{t}$ and $\delta$ be as in Proposition 4.1. Define $h_{e}: X_{1} \times I \rightarrow C\left(X_{1}\right)$ by

$$
h_{e}(p, t)=h(\{p\}, t \bar{t}) \cap X_{1} .
$$

4.3. Theorem. If $C(X)$ is contractible then either $X_{1}$ or $X_{2}$ is contractible im kleinen at $p_{0}$.

\section{The characterization theorem.}

5.1. Theorem. $C(X)$ is contractible if and only if $C\left(X_{1}\right)$ and $C\left(X_{2}\right)$ are both contractible and either $X_{1}$ or $X_{2}$ is contractible im kleinen at $p_{0}$.

Proof. We need only prove sufficiency. Suppose $X_{1}$ is contractible im kleinen at $p_{0}$. By Lemma 3.7, there is a deformation retract $h_{0}: C\left(X_{1}\right) \times I \rightarrow C\left(X_{1}\right)$ of $C\left(X_{1}\right)$ onto $\mathscr{F}_{1}$. Denote by $h_{1}$ and $h_{2}$ monotone contractions of $C\left(X_{1}\right)$ and $C\left(X_{2}\right)$. Define $h: C(X) \times I \rightarrow C(X)$ by first deforming $C(X)=C\left(X_{1}\right) \cup\left(\mathscr{F}_{1} \times \mathscr{F}_{2}\right) \cup C\left(X_{2}\right)$ onto $\left(\mathscr{F}_{1} \times \mathscr{F}_{2}\right) \cup C\left(X_{2}\right)$ by means of $h_{0}$; second, deforming $\left(\mathscr{F}_{1} \times \mathscr{F}_{2}\right) \cup C\left(X_{2}\right)$ onto $\mathscr{F}_{1} \times\left\{X_{2}\right\}$ by means of $h_{2}$; finally, deforming $\mathscr{F}_{1} \times\left\{X_{2}\right\}$ to $\left\{X_{1}\right\} \times\left\{X_{2}\right\}=$ $X$ by means of $h_{1}$.

5.2. TheOREM. Suppose $X_{1}$ and $X_{2}$ both have property $K$. Then $C(X)$ is contractible when and only when either $X_{1}$ or $X_{2}$ is connected im kleinen at $p_{0}$.

Proof. The theorem follows from Theorems 3.4 and 5.1. 
5.3. ExAmple. We give the example of [2]. Let $Y$ be the closure in the plane of $\{(u, v) \mid v=\sin (1 / u)$ for some $0<u<1\}$. One can easily show $Y$ has property $K$. Let $X_{1}$ and $X_{2}$ be two copies of $Y$ and $X=X_{1} \cup X_{2}$ with $\left\{p_{0}\right\}=X_{1} \cap X_{2}$ such that neither $X_{1}$ nor $X_{2}$ is connected im kleinen at $p_{0}$. Then $C(X)$ is not contractible, a fact already observed by Kelley in [2].

We have the following two consequences of Theorem 5.1.

5.4. Proposition. If $C\left(X_{1}\right)$ and $C\left(X_{2}\right)$ are contractible and there are arbitrarily small subcontinua $X_{\varepsilon}$ of $X_{1}$ whose interior relative to $X_{1}$ contains $p_{0}$ and $C\left(X_{\varepsilon}\right)$ are contractible, then $C(X)$ is contractible.

5.5. Proposition. If $C\left(X_{1}\right)$ and $C\left(X_{2}\right)$ are contractible and $X_{1}$ has arbitrarily small contractible subcontinua $X_{e}$ whose interiors relative to $X_{1}$ contains $p_{0}$, then $C(X)$ is contractible.

5.6. ExAmple. Let $X_{1}$ be the arc $I$ and $X_{2}$ be a pseudo arc such that $X_{1} \cap X_{2}=$ $\left\{p_{0}\right\}$ and $X=X_{1} \cup X_{2}$. Then $C(X)$ is contractible.

Proof. By [5], $C\left(X_{2}\right)$ is contractible. See also [3].

6. Remark. There is a continuum $Y$ which is connected im kleinen at $q_{0}$ but not contractible im kleinen. Let $Y_{n}$ be the closure in the plane of the set $\{(u, v+2 n) \mid v$ $=\sin (1 / u)$ for some $0<u \leqslant 1\}$ and let $Y$ be the one-point compactification of $\cup_{n=0}^{\infty} Y_{n}$. Then one sees that $Y$ is connected im kleinen at $\infty$, but not contractible im kleinen at $\infty$. The latter fact can be proved by using techniques from $\$ 4$ and the fact that $Y$ is not connected im kleinen at each cut point of $Y$ whose first coordinate is zero.

\section{REFERENCES}

1. J. G. Hocking and G. S. Young, Topology, Addison-Wesley, Reading, Mass., 1961.

2. J. L. Kelley, Hyperspaces of a contimum, Trans. Amer. Math. Soc. 52 (1942), 22-36.

3. J. Krasinkiewicz, On the hyperspaces of hereditarily indecomposable continua, Fund. Math. 84 (1974), 175-186.

4. S. B. Nadler, Jr., Hyperspaces of sets, Dekker, New York, 1978.

5. C. J. Rhee, On dimension of hyperspace of a metric continumm, Bull. Soc. Roy. Sci. Liege 38 (1969), 602-604.

6. G. T. Whyburn, Analytic topology, Amer. Math. Soc. Colloq. Publ., vol. 28, Amer. Math. Soc., Providence, R.I., 1942.

Department of Mathematics, Wayne State University, Detrort, Michigan 48202 